Saudi Journal of Medicine

Abbreviated Key Title: Saudi J Med ISSN 2518-3389 (Print) |ISSN 2518-3397 (Online)

\title{
Knowledge of Efficacy of Guided Implant Surgery in Prosthodontics amongst Implantologists - A Qualitative Research
}

Dr. Digvijaysinh Parmar, BDS, MPH ${ }^{1 *}$, Dr. Priya Shah, BDS ${ }^{2}$, Dr. Rashmi Kolichala, BDS, MPA ${ }^{3}$, Dr. Harshitha Garlapati, BDS $^{4}$, Dr. Nuba Afzal Siddiqi, BDS ${ }^{5}$, Dr. Aiman Tauqeer, BDS ${ }^{6}$

${ }^{1}$ PMNM Dental College and Hospital, Karnataka, India

${ }^{2}$ Darshan Dental College and Hospital, Loyara, Udaipur, India

${ }^{3}$ Sri. Venkata Sai Institute of Dental Sciences, Telangana, India

${ }^{4}$ Narayana Dental College and Hospital, Nellore, Andhra Pradesh, India

${ }^{5}$ Uttar Pradesh Dental College, Lucknow, India

${ }^{6}$ San Antonio, United states

DOI: $10.36348 /$ sim.2020.v05i04.007

| Received: 02.04.2020 | Accepted: 14.04.2020 | Published: 15.04.2020

*Corresponding author: Dr. Digvijaysinh Parmar

\section{Abstract}

Aim of the study: The purpose of this study was to assess the choices and viewpoints of various Implantologists regarding efficacy of guided implant surgery in the field of prosthodontics. Methodology: A questionnaire survey was conducted amongst 10 Implantologists over a period of 2 years about the efficacy of guided implant surgery for prosthetic rehabilitation. Results: The results show that Implantologists prefer both static and dynamic computer aided navigation placement of Implants. Dynamic was preferred in cases more (69\%), where there was reduced mouth opening as opposed to static navigation systems especially in the posterior region of the oral cavity. Conclusion: Guided implants insertion often permits mini-invasive surgeries without the need to elevate a surgical flap. Computer guided implant surgery may appear to provide dental professionals with a all-inclusive, reliable and detailed solution to sophisticated cases bringing about novel and exciting possibilities.

Keywords Guided implant surgery, CAD-CAM, less invasive, precision.

Copyright @ 2020: This is an open-access article distributed under the terms of the Creative Commons Attribution license which permits unrestricted use, distribution, and reproduction in any medium for non-commercial use (NonCommercial, or CC-BY-NC) provided the original author and source are credited.

\section{INTRODUCTION}

Digital workflow has increasingly had an imperative role to play in contemporary dentistry. The advantage of guided implant surgery is that the implant is placed in a innocuous, more foreseeable manner, via a surgical template designed and shaped using computer aided- design/computer-aided-manufacturing (CAD/CAM) technology; this prosthetically guided placement is achieved using software for virtual implant planning. Guided implant surgery can also aid the dentist to accomplish flapless implant surgery with less distress for the patient and faster working and healing times as compared to conventional implant placement [1] (Table 1). Over the years, many resolutions have been proposed in order to progress the clinical performance of dental implants. Implant shape has changed with the introduction of not only cylindrical structures, of most effectual coil design and of better implant prosthetic connections. Many surface treatments have also been recommended to modify the nanostructure of titanium, refining Osseo integration processes and bone healing [2]. Guided implant surgery (GIS) allows allocating planned reintegration project straight into surgical field. The clinician can choose between several guided methods; first, surgical guides can be divided into "static" and "dynamic". The latter are characterized by guided navigation methods in which a computer-guided navigation system aids the clinician in real time during the implant positioning through visual imaging tools on a monitor. These methods, though very interesting in future viewpoint are currently not mainly widespread [3]. Digital scanning and Cone Beam Computer Tomography (CBCT) are the measures now used for digital workflow for development of guided implant surgery [4]. Considering optical imprints with prevailing intraoral scanners for fabricating permanent prostheses on natural teeth and dental implants is becoming extensive and has many advantages over the conventional way of taking impressions, involving less uneasiness for the patient, as well as greater speed, precision, precision, and reproducibility[5]. Optical impression-taking allows assemblage of all the threedimensional (3D) information of dento-gingival tissues [6]. CBCT on the other hand allows assortment all 3D data on the anatomy of the residual crest bone, 
including height, thickness, and angle [7]. Guided implants insertion often permits mini-invasive surgeries without the need to elevate a surgical flap. An additional benefit of guided techniques is to have, at the time of surgery, a prefabricated fixed prosthesis, built on planned implants position, able to join newly inserted implants and to easily accomplish a functional and aesthetic immediate loading[8]. The composition and superimposition of dental and gingival information acquired by intraoral scanning, as well as bone information acquired by CBCT, now allow virtual planning for placing the implants, fabricating the templates for guided surgery, and modelling and preparing temporaries for immediate loading [9].

\section{AIM OF THE STUDY}

The purpose of this study was to assess the choices and viewpoints of various Implantologists regarding efficacy of guided implant surgery in the field of prosthodontics.

\section{METHODOLOGY}

A questionnaire survey was conducted amongst 10 Implantologists over a period of 2 years about the efficacy as well as precision of guided implant surgery for prosthetic rehabilitation as well as their take on survival rates of conventional versus guided implant placement. E-mail was sent in an openended format in English language. Their responses were recorded on a spreadsheet and was analyzed by descriptive statistics

\section{RESULTS}

The results show that Implantologists prefer both static and dynamic computer aided navigation placement of Implants. Dynamic was preferred in cases more $(69 \%)$, where there was reduced mouth opening as opposed to static navigation systems especially in the posterior region of the oral cavity (Table 2). Computeraided static navigation techniques are achieved using surgical templates to avert the necessity for drilling guidance during surgery. Consequently, implant placement precision rest on directly the design and manufacturing process of the surgical template; if there is an imprecision during the fabrication process, this might lead to intraoperative difficulties. On the other hand, computer-aided dynamic navigation systems permit for a straight view of the surgical field and deliver clinicians with the ability to delocalize the position of an implant, if necessary. Foremost disadvantage of computer-aided dynamic navigation systems is the struggle in keeping sight of the dynamic navigation system display during the surgical procedure. Though, augmented reality devices can be used to display the virtual image of the computer-aided dynamic navigation system without trailing sight of the surgical field [10]. Most Implantologists thought that there are few chief indications for guided implant surgery for prosthetic rehabilitation where use of CBCT can be of much importance (Table 3). Rehabilitations in mandible with vertical bone deficiency $(46.7 \%)$ had most of clinicians in its favour whereas other situations like Fixed rehabilitations in edentulous maxilla with atrophy $(35 \%)$ and Single tooth replacements in aesthetic zone were the other considerations for using guided implant surgery. (18.3\%)

Table-1: Disadvantages with conventional implant placement

\begin{tabular}{|l|}
\hline Problems with conventional implant placement \\
\hline Loss of tissue support (Bone/Soft tissue) \\
\hline May not have ideal implant positions \\
\hline Chances of implant failure increases \\
\hline More invasive with incision and flap eversion \\
\hline Increased healing time \\
\hline Compromising primary stability \\
\hline Compromising immediate loading restorations techniques \\
\hline
\end{tabular}

Table-2: Static versus Dynamic computer aided navigation of implant placement

\begin{tabular}{|l|l|l|}
\hline Guided implant placement areas/situations & Static navigation & Dynamic navigation \\
\hline Reduced mouth opening & $31 \%$ & $69 \%$ \\
\hline Anterior region & $55 \%$ & $45 \%$ \\
\hline Posterior region & $40.4 \%$ & $59.6 \%$ \\
\hline
\end{tabular}

Table-3: Choices where guided implant surgery was preferred according to Implantologists

\begin{tabular}{|l|l|}
\hline Single tooth replacements in aesthetic zone & $\mathbf{1 8 . 3 \%}$ \\
\hline Fixed rehabilitations in edentulous maxilla with atrophy & $35 \%$ \\
\hline Rehabilitations in mandible with vertical bone deficiency & $46.7 \%$ \\
\hline
\end{tabular}

\section{DISCUSSION}

Digital implantology is one of the most imperious innovation drivers in dentistry and the pace keeps escalating. Computer guided implant surgery is dependent on comprehensive diagnostics and precise planning to permit stringent reverse prosthetic driven planning in order to achieve optimised surgical and prosthetic outcome. While improving the safety and 
efficiency of the surgical procedure computer guided implant surgery renders the restorative outcome more predictable regarding function, biology and aesthetics [11]. Precise one-to-one measurements of the width and height of bone in planned implant sites, as well as distances and angulations between implants from one side of an arch to another, can be programmed without the distortions that are present in the two-dimensional radiology [12]. Implants and abutments can then be "virtually" premeditated, driven by knowledge of the position of the strategic restoration. It also permits predetermination of prosthesis path of insertion, assignment of screw chambers, componentry space, and pre-surgical abutment choices, as well as pre-surgical fabrication of individual abutments. A precise virtual surgery planning lets sometimes to evade bone augmentation procedures which are associated to an extension of treatment time. Farley et al., [13] in a splitmouth, randomized controlled trial, described that computer-assisted, template-based implant placement was further precise than conventional guides, but only for coronal horizontal distances. One in vitro comparative study (80 implants) stated that the 3-D accurateness of single-tooth implants placed using piloted implant surgery compared with conventional implant placement. Kramer et al. compared in vitro the precision of conventional $(n=40)$ versus navigated $(n=$ 40) implant placement. For each group, identical maxillary casts were used to place implants for singletooth replacement of either the left central incisor $(\mathrm{n}=$ $20)$ or the right canine $(n=20)$. The authors determined that variation in implant position, angulation and penetration was reduced for implants that were placed using the navigation protocol. In a prospective study, Behneke et al. analysed the issues that may influence the transmission precision of CBCT-derived, laboratory-based surgical guides for implant placement in partially edentulous patients. Nineteen implants were positioned to reinstate a single-tooth gap in 19 partially edentulous patients. The precision of computer-assisted, template-based implant placement was assessed using the image fusion technique. Measurements were completed to calculate linear and angular deviations amid virtually planned and actually placed implants with template guidance in both single-tooth gap and abridged residual dentition cases. A mean error of 0.21 $\pm 0.16 \mathrm{~mm}$ (range of $0.01-0.92 \mathrm{~mm}$ ) at the entry point and of $0.32 \pm 0.34 \mathrm{~mm}$ (range of $0.03-0.59$ ) at the apex, and $1.35 \pm 1.11^{\circ}$ (range of $0.07-3.33^{\circ}$ ) of apex radial deviation were stated for single-tooth gap surgery[14]. Implantologists these days prefer guided implant surgery these days over conventional implant placement. Improved accuracy was noted, however, when the implant was placed fully guided (i.e., all drill steps and implant placement occur through the guide), as compared to freehand placement of the implant, with all drilling done guided, and when the final drill sequence was freehand and only the first drill(s) guided[15].

\section{CONCLUSION}

Marginally invasive techniques appeal to a larger number of potential implant patients and is also often accompanied with condensed total rehabilitation time and economic benefits. Computer guided implant surgery may appear to provide dental professionals with a all-inclusive, reliable and detailed solution to sophisticated cases bringing about novel and exciting possibilities.

\section{REFERENCES}

1. Dolcini, G. A., Colombo, M., \& Mangano, C. (2016). From guided surgery to final prosthesis with a fully digital procedure: a prospective clinical study on 15 partially edentulous patients. International journal of dentistry, 2016. Smeets, R., Stadlinger, B., Schwarz, F., BeckBroichsitter, B., Jung, O., Precht, C., ... \& Ebker, T. (2016). Impact of dental implant surface modifications on osseointegration. BioMed Research International, 2016.

2. Colombo, M., Mangano, C., Mijiritsky, E., Krebs, M., Hauschild, U., \& Fortin, T. (2017). Clinical applications and effectiveness of guided implant surgery: a critical review based on randomized controlled trials. BMC Oral Health, 17(1), 150.

3. Van der Meer, W. J., Andriessen, F. S., Wismeijer, D., \& Ren, Y. (2012). Application of intra-oral dental scanners in the digital workflow of implantology. PloS one, 7(8).

4. Zimmermann, M., Mehl, A., Mörmann, W. H., \& Reich, S. (2015). Intraoral scanning systems-a current overview. International journal of computerized dentistry, 18(2), 101-129.

5. Yuzbasioglu, E., Kurt, H., Turunc, R., \& Bilir, H. (2014). Comparison of digital and conventional impression techniques: evaluation of patients' perception, treatment comfort, effectiveness and clinical outcomes. BMC oral health, 14(1), 10.

6. Arunyanak, S. P., Harris, B. T., Grant, G. T., Morton, D., \& Lin, W. S. (2016). Digital approach to planning computer-guided surgery and immediate provisionalization in a partially edentulous patient. The Journal of prosthetic dentistry, 116(1), 8-14.

7. van Steenberghe, D., Glauser, R., Blombäck, U., Andersson, M., Schutyser, F., Pettersson, A., \& Wendelhag, I. (2005). A computed tomographic scan-derived customized surgical template and fixed prosthesis for flapless surgery and immediate loading of implants in fully edentulous maxillae: A prospective multicenter study. Clinical implant dentistry and related research, 7, s111-s120.

8. Lee, J. H. (2016). Intraoral digital impression for fabricating a replica of an implant-supported interim prosthesis. The Journal of prosthetic dentistry, 115(2), 145-149.

9. Mediavilla Guzmán, A., Riad Deglow, E., Zubizarreta-Macho, Á., Agustín-Panadero, R., \& Hernández Montero, S. (2019). Accuracy of 
Computer-Aided Dynamic Navigation Compared to Computer-Aided Static Navigation for Dental Implant Placement: An In Vitro Study. Journal of Clinical Medicine, 8(12), 2123.

10. Zayed, S. M. (2019). Computer Guided Implant Surgery: Is It a Holistic Solution?. EC Dental Science, 18, 1302-1312.

11. Van Steenberghe, D., Ericsson, I., Van Cleynenbreugel, J., Schutyser, F., Brajnovic, I., \& Andersson, M. (2004). High precision planning for oral implants based on 3-D CT scanning. A new surgical technique for immediate and delayed loading. Applied osseointegration research, 4, 2731.

12. Lindeboom, J. A., \& Van Wijk, A. J. (2010). A comparison of two implant techniques on patientbased outcome measures: a report of flapless vs. conventional flapped implant placement. Clinical oral implants research, 21(4), 366-370.

13. Tallarico, M., Meloni, S. M., Canullo, L., Xhanari, E. P. G., \& Polizzi, G. (2016). Guided surgery for single-implant placement: A critical review. J Oral Sci Rehabil [internet].

14. Anders, N. (2017). Image-Guided Implant Surgery. Decisions in Dentistry. December. 3(12):11-15. 